\title{
Falla de sutura en cirugía colorrectal. Factores de riesgo y manejo terapéutico
}

\author{
Javier Chinelli ${ }^{1}$, Juan Costa $^{1}$, Emilia Moreira ${ }^{2}$, Gustavo Rodríguez ${ }^{1}$ \\ ${ }^{1}$ Cirujano \\ ${ }^{2}$ Residente de Cirugía General Clínica Quirúrgica 2. Hospital Maciel. Montevideo, Uruguay.
}

\begin{abstract}
RESUMEN
Introducción: La dehiscencia anastomótica (DA) es una complicación de la cirugía colorrectal que determina alta morbimortalidad y peor pronóstico oncológico. El objetivo de este estudio es analizar su relación con factores de riesgo y describir su manejo terapéutico.

Métodos: Estudio retrospectivo, observacional y analítico. Se incluyeron resecciones colorrectales de coordinación por patología neoplásica maligna con anastomosis primaria, entre enero de 2015 y noviembre de 2018.

Resultados: Ciento doce pacientes cumplieron con los criterios de inclusión. La tasa de DA fue $17 \%$ y la mortalidad $15 \%$. El tamaño tumoral se asoció de forma estadísticamente significativa con un mayor riesgo de falla de sutura.

Discusión: Nuestros resultados son similares a los reportados en la literatura, destacándose una alta proporción de DA tempranas y por lo tanto de reintervención y detransitación.

Conclusiones: El manejo de la DA es un reto terapéutico y debe ajustarse a muchos parámetros, siendo uno de los más importantes la experiencia del cirujano.
\end{abstract}

Palabras clave: Cirugía Colorrectal; Dehiscencia Anastomótica; Factores de Riesgo

\section{SUMMARY}

Introduction: Anastomotic dehiscence $(A D)$ is a complication of colorectal surgery that determines high morbidity and mortality and a worse oncological prognosis. The objective of this study is to analyze its relationship with risk factors and describe its therapeutic management.

Material and Methods: Retrospective, observational and analytical study. Colorectal resections with primary anastomosis for malignant neoplastic pathology performed between January 2015 and November 2018 were included.

Results: One hundred and twelve patients met the inclusion criteria. The AD rate was $17 \%$ and mortality $15 \%$. Tumor size was statistically significantly associated with an increased risk of $A F$.

Discussion: Our results are similar to those reported in the literature, highlighting a high proportion of early $A D$ and therefore of re-intervention and intestinal diversion.

Conclusions: The management of $A D$ is a therapeutic challenge, and must be adjusted to many parameters, one of the most important being the surgeon's experience.

Keywords: Colorectal Surgery; Anastomotic Dehiscence; Risk factors

\section{INTRODUCCIÓN}

Las complicaciones quirúrgicas son eventos inherentes al tratamiento de algunas patologías con procedimientos más o menos invasivos. La importancia de este tema abarca múltiples aspectos, entre ellos los relacionados al aumento de los costos sanitarios durante el proceso asistencial. $^{1}$

La cirugía colorrectal tiene una morbilidad que oscila entre un 10 y un $30 \% .^{2}$

Entre las distintas complicaciones, la falla de sutura tiene un rol protagónico por su potencial gravedad, siendo responsable de hasta un tercio de las muertes postoperatorias. $^{3}$ Además, hay evidencia de que la dehiscencia anastomótica también es un factor de mal pronóstico oncológico a largo plazo. ${ }^{4}$

Su incidencia global de acuerdo a lo señalado en la li-

Los autores declaran ausencia de conflictos de interés.

Javier Chinelli

jchinelli01@gmail.com

Recibido: febrero de 2020. Aceptado: marzo de 2020 teratura oscila entre 3-6\%, ${ }^{5}$ aunque también resulta ser muy variable, ya que depende del tipo y nivel de anastomosis que se considere, ${ }^{6}$ así como de las múltiples definiciones que existen y en consecuencia de la falta de estandarización en su diagnóstico. ${ }^{7}$ Varios factores de riesgo han sido estudiados como predictores de complicaciones en general para la cirugía colorrectal, ${ }^{8}$ pero sobre todo se ha intentado establecer cuáles son los que inciden en la aparición de una falla de sutura. Entre estos últimos se destacan el sexo masculino, estado nutricional, anemia, el tamaño tumoral, la ubicación por debajo de $\operatorname{los} 12 \mathrm{~cm}$ respecto al margen anal, una anastomosis por debajo de los $10 \mathrm{~cm}$, tiempo operatorio prolongado, sangrado intraoperatorio, obesidad y radioterapia pelviana. ${ }^{9-11}$

Por otra parte, el tratamiento integral de esta complicación sigue siendo un desafío, contando actualmente con un amplio arsenal terapéutico, sea farmacológico, percutáneo, endoscópico, quirúrgico o la combinación de estos, siendo aquí donde suelen prevalecer el juicio y la experiencia del cirujano actuante. Es importante reconocer también que al evaluar el tratamiento de una complicación de 
incidencia relativamente baja como la falla de sutura, la evidencia surge necesariamente de estudios retrospectivos de grandes series de pacientes, dada la dificultad que supone diseñar estudios prospectivos con esta finalidad. ${ }^{12}$

El objetivo de este trabajo es determinar el rol de diversos factores de riesgo de falla de sutura y describir el manejo terapéutico de la misma en la población estudiada.

\section{PACIENTES Y MÉTODOS}

\section{Estudio multicéntrico, observacional, retrospectivo y analítico}

Se incluyeron todos los pacientes operados de coordinación a los que se les realizó una resección de colon y/o recto con anastomosis primaria, por patología neoplásica maligna, en el período enero de 2015-noviembre de 2018, en la Clínica Quirúrgica 2 (Hospital Maciel) y Corporación Médica de Canelones (COMECA). Se excluyeron otros procedimientos como cierre de colostomía o restitución del tránsito, dado que en esos casos no es posible evaluar algunos de los factores de riesgo. Se definió a la falla de sutura por la presencia de elementos clínicos (síndrome de respuesta inflamatoria sistémica, peritonitis), imagenológicos (líquido libre intraperitoneal, neumoperitoneo, colección perianastomótica con o sin gas) e intraoperatorios (constatación de la dehiscencia a nivel de la sutura).

Los datos se obtuvieron a partir de las historias clínicas de los pacientes. Se consignaron las siguientes variables: edad (en años), sexo, cirugía abdominal previa, insuficiencia renal (Creatininemia $>1,2 \mathrm{mg} / \mathrm{dl}$ ), insuficiencia cardíaca, topografía del tumor, tamaño tumoral $(\mathrm{en} \mathrm{cm})$, hipoalbuminemia preoperatoria $(<3,5 \mathrm{mg} / \mathrm{dl})$, neoadyuvancia, anemia preoperatoria $(\mathrm{Hb}<10 \mathrm{mg} / \mathrm{dl})$, abordaje laparoscópico o abierto, tiempo operatorio (en minutos), tipo de anastomosis (manual o mecánica), altura de la anastomosis (intra o subperitoneal), detransitación de anastomosis, colocación de drenajes, experiencia del cirujano (senior vs junior), falla de sutura: precoz (antes del sexto día) o tardía, manejo terapéutico de la misma y mortalidad (tomando como punto de corte el momento de la recolección de datos).

Las variables cuantitativas se expresan en media y desvío estándar (DE) y las variables cualitativas en frecuencia relativa porcentual. Por tratarse de un estudio retrospectivo se estimó el riesgo de cada variable mediante Odds Ratio. Aquellas que resultaron en un OR significativo individualmente se incluyeron en la conformación de un modelo multivariado, considerándose como variables de aumento del riesgo las que siguieron siéndolo en dicho modelo. Los datos se procesaron mediante software estadístico SPSS IBM (versión 22.0). Se acepta un valor de $\mathrm{p}$ $<0,05$ como estadísticamente significativo.

\section{Aspectos éticos}

La participación de los pacientes fue anónima. Por tratarse de un estudio retrospectivo no implica una interferencia en el proceso asistencial del paciente ni existe riesgo para los sujetos de estudio, ya que no estuvieron expuestos a ningún cambio de conducta. La información fue procesada solamente por los autores de la investigación, respetando el secreto profesional, y se asignó a cada paciente un número autogenerado con el fin de preservar la confidencialidad.

No se solicitó consentimiento informado por tratarse de un estudio observacional en el cual se utilizaron datos de forma anónima y con fines estadísticos.

El trabajo fue aprobado por el comité de ética hospitalario.

\section{RESULTADOS}

Un total de 112 pacientes (56 en cada institución que participó del estudio) cumplieron con los criterios de inclusión, $49(43,8 \%)$ de sexo femenino y $63(56,2 \%)$ masculino, con una media de edad de 66,6 años $(30-89$, DE 11,746). El tamaño promedio de las lesiones fue de $4,4 \mathrm{~cm}$ (0,7-16,1, DE 2,5192), y el tiempo operatorio medio 160,7 minutos $(60-390, \mathrm{DE} 60,940)$. En un 79,5\% de los pacientes el abordaje fue laparoscópico y en el 20,5\% abierto (tabla 1).

La distribución porcentual de los tumores de acuerdo a su topografía fue la siguiente: colon ascendente $33,9 \%$, transverso 5,4\%, ángulo izquierdo $1,8 \%$, descendente $2,7 \%$, sigmoides $22,3 \%$, recto $33,9 \%$ (graf. 1 ).

De acuerdo a la experiencia del cirujano actuante, 59 procedimientos fueron realizados por cirujano junior y 53 de ellos por cirujano senior.

En el 68\% de los casos la anastomosis fue mecánica, y en el $80,4 \%$ la misma quedó localizada a nivel intraperitoneal. La detransitación de anastomosis se utilizó en el $10,7 \%$ de los pacientes y el uso de drenajes en un $46,4 \%$.

Diecinueve pacientes presentaron una falla de sutura (17\%). Si consideramos la falla de sutura de acuerdo al tipo de anastomosis realizada, la distribución fue la siguiente: ileocólica 20\% (10/50); colorrectal intraperitoneal 10,4\% (5/48); colorrectal subperitoneal 22,2\% (4/18).

\section{La mortalidad global fue del 15,2\%.}

Se calculó el riesgo de cada uno de esos factores para fa1la de sutura con su intervalo de confianza (IC) de 95\%, cuyos resultados se resumen en la tabla 2 .

También se calculó el riesgo de alguno de estos factores en relación a la mortalidad (tabla 3).

De acuerdo al momento de presentación y manejo terapéutico de la falla de sutura, en 13 pacientes el diagnóstico fue en etapa precoz (antes del sexto día), mientras que 
en 6 fue en etapa tardía.

El tratamiento de acuerdo al tipo de falla fue el siguiente:

- Falla de sutura ileocólica (10): desmontaje e ileostomía en 7 , antibioticoterapia en 1 , drenaje percutáneo en 1 y abstención terapéutica en 1 (estadio IV).

- Falla de sutura colorrectal (9): Desmontaje y Hartmann en 8 y drenaje percutáneo transglúteo en 1.

\section{DISCUSIÓN}

En la población de estudio la tasa global de dehiscencia anastomótica fue de $17 \%$, con una presentación temprana en la mayoría de los casos.

La incidencia de falla de sutura en cirugía colorrectal es variable, existiendo estudios que informan tasas de 3 a $28 \%,{ }^{13}$ en tanto que series de centros de alto volumen reportan cifras algo menores, entre 1,6-9,9\%. ${ }^{14}$ Teniendo en cuenta estos datos, los resultados de nuestra serie se encuentran dentro de lo señalado en la bibliografía, aunque se acercan al límite superior de lo habitualmente aceptado. El riesgo de dehiscencia anastomótica aumenta cuanto más baja es la sutura. ${ }^{6}$ Si bien, en nuestra serie, y en concordancia con lo anteriormente señalado, el mayor porcentaje de fallas se da entre las anastomosis colorrectales subperitoneales $(22,2 \%)$, existió un porcentaje apenas inferior de falla de anastomosis ileocólicas (20\%), lo que quizás se explique por la baja potencia del estudio. No obstante, debemos señalar algunas falencias de las investigaciones que constituyen la fuente actual de evidencia. En primer lugar, la definición de lo que se considera como "fuga anastomótica", que puede ser extremadamente variable en términos de etapa de presentación clínica, traducción imagenológica y/o hallazgos intraoperatorios como por ejemplo el grado o extensión de la dehiscencia a nivel de la línea de sutura, lo que sin dudas determina la falta de una definición estandarizada. ${ }^{15}$ De hecho, una revisión sistemática sobre 97 estudios $^{16}$ encontró un total de 56 definiciones distintas para la falla de sutura, demostrando así claramente el alcance y la complejidad del problema al que hacemos referencia. Nuestro trabajo no excluye de la definición de falla de sutura aquellos casos en los que la presentación fue tardía, en general a forma de absceso, independientemente del tratamiento instituido, sabiendo que de no hacerlo estaríamos subestimando la verdadera incidencia de esta complicación. En 2010, el International Study Group of Rectal Cancer, en un intento por unificar criterios diagnósticos y terapéuticos, define a la falla de sutura como cualquier defecto a nivel de la anastomosis (incluyendo las líneas de sutura de los reservorios rectales) que produzca una comunicación entre los compartimentos endoluminal y extraluminal. ${ }^{17}$ En se-
TABLA 1: DATOS DEMOGRÁFICOS, TAMAÑO TUMORAL, TIEMPO OPERATORIO Y VÍA DE ABORDAJE

\begin{tabular}{|c|c|}
\hline $\mathrm{n}$ total & 112 \\
\hline Sexo femenino, n (\%) & $49(43,8)$ \\
\hline Sexo masculino, $\mathrm{n}(\%)$ & $63(53,2)$ \\
\hline Edad (años), promedio \pm DS & $66,6 \pm 11,74$ \\
\hline Tamaño tumoral (cm), promedio \pm DS & $4,4 \pm 2,51$ \\
\hline $\begin{array}{l}\text { Tiempo operatorio ( } \mathrm{min} \text { ), } \\
\text { promedio } \pm \mathrm{DS}\end{array}$ & $160,7 \pm 60,94$ \\
\hline Abordaje laparoscópico & $79,50 \%$ \\
\hline Abordaje abierto & $20,50 \%$ \\
\hline
\end{tabular}

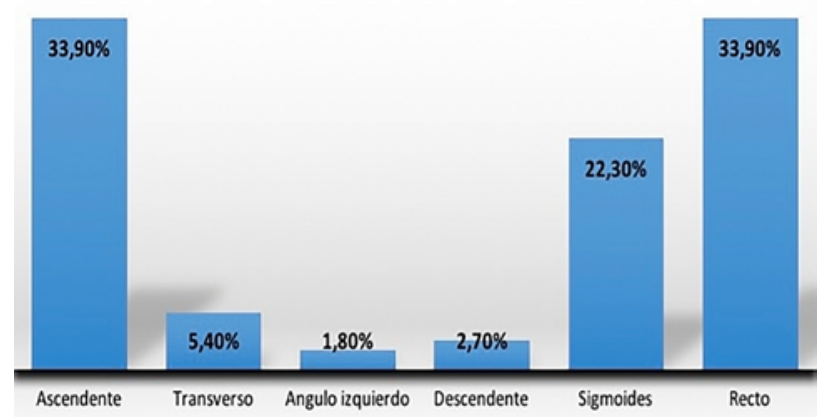

Gráfico 1: Indicaciones de sigmoidectomía electiva por enfermedad diverticular.

gundo lugar, la tasa de dehiscencia anastomótica también varía según el tipo de anastomosis que se considere (ileocólica, colocólica, ileoanal), ${ }^{18}$ las que invariablemente suelen coexistir en la mayoría de los trabajos que investigan el problema, como sucede en el nuestro.

Actualmente se reconocen dos patrones de falla de sutura según el momento de presentación: una precoz, en general antes del sexto día postoperatorio y otra tardía, posterior a este. Las fallas precoces suelen estar asociadas a factores de riesgo que traducen un procedimiento dificultoso, especialmente al momento de confeccionar la anastomosis (sangrado intraoperatorio, obesidad, tiempo operatorio prolongado), ${ }^{19}$ en tanto que las fallas tardías se asocian a diversos factores que de alguna forma afectan los procesos de reparación y cicatrización tisular (neoadyuvancia, hipoalbuminemia, etc.). ${ }^{20,21}$ En nuestra serie la mayoría de las fallas ocurrieron en una etapa precoz, y la forma de presentación en esos casos fue la peritonitis aguda. En cambio, la presentación tardía fue fundamentalmente bajo la forma de abscesos intraabdominales. Sin embargo, no todas las investigaciones coinciden en sus resultados al intentar determinar la asociación y/o la magnitud de factores de riesgo con la falla de sutura. ${ }^{22}$ Entre ellos se destacan: el sexo masculino, el índice de masa corporal (IMC), anemia/hipoalbuminemia, tratamiento 
TABLA 2: FACTORES DE RIESGO Y FALLA DE SUTURA

\begin{tabular}{lccc}
\hline Factor & Odds Ratio (OR) & Intervalo de confianza de $95 \%$ & $p$ \\
\hline Sexo & 0,825 & $0,319-2,137$ & \\
\hline Cirugía previa & 1,280 & $0,438-3,738$ & $0,314-3,574$ \\
\hline Insuficiencia renal & 1,059 & $0,460-8,286$ & \\
\hline Insuficiencia cardiaca & 1,952 & $0,150-2,127$ & \\
\hline Hipoalbuminemia & 0,565 & $0,122-10,162$ & \\
\hline Neoadyuvancia & 1,113 & $0,369-3,026$ & \\
\hline Anemia & 1,056 & $0,338-3,727$ & \\
\hline Abordaje & 1,122 & $0,269-1,938$ & 0,764 \\
\hline Anastomosis (manual/mecánica) & 0,722 & $0,428-4,228$ & 0,045 \\
\hline Altura de la anastomosis & 1,360 & $0,057-3,913$ & 0,336 \\
\hline Detransitación & 0,472 & $0,410-2,743$ & 4,201 \\
\hline Drenaje & 1,061 & & \\
\hline Edad & 0,994 & & \\
\hline Tamaño tumoral & 1,018 & 0,601 & \\
\hline Tiempo operatorio & 1,004 & & 0,313 \\
\hline Experiencia (junior / senior) & 1,590 & & 0,368 \\
\hline Topografía & & & \\
\hline Ascendente & 0,226 & & \\
\hline Transverso & 0,200 & & \\
\hline Angulo izquierdo & & & \\
\hline Descendente & 0,500 & & \\
\hline Sigmoides & 0,250 & & \\
\hline Recto & 0,188 & & \\
\hline
\end{tabular}

neoadyuvante, número de disparos de máquina de sutura, ostomía de detransitación, tiempo operatorio prolongado, uso de drenajes, la preparación colónica preoperatoria, altura de la anastomosis a menos de $10 \mathrm{~cm}$ del margen anal y la experiencia del cirujano/volumen anual de la institución. ${ }^{23-29}$ Uno de los objetivos de este estudio fue analizar la asociación entre algunos de los factores de riesgo y la falla de sutura principalmente, así como con la mortalidad. Como puede verse en las tablas 2 y 3 , solamente el tamaño tumoral se asoció de forma estadísticamente significativa con el riesgo de falla de sutura (OR 1,018; $\mathrm{p}=0,045)$. De la misma forma, solamente la topografía en ángulo izquierdo y la insuficiencia cardíaca (OR 7,333, IC 95\% 1,848-29,107) se asociaron en forma estadísticamente significativa a la mortalidad. Pese a ello, debemos tener en cuenta el pequeño tamaño muestral de nuestra serie, por lo que la baja potencia del estudio debe conducir a una cuidadosa interpretación de los resultados obtenidos. En ese mismo sentido, es de suponer que el factor experiencia del cirujano actuante/volumen anual de la institución incida en los resultados aún más de lo que hemos podido demostrar en nuestra población de estudio. A esto debe
TABLA 3: FACTORES DE RIESGO Y MORTALIDAD

\begin{tabular}{lcc} 
Factor de riesgo & $\begin{array}{c}\text { Odds Ratio } \\
\text { (OR) }\end{array}$ & $\begin{array}{c}\text { Intervalo de } \\
\text { confianza de } \\
95 \%\end{array}$ \\
\hline Sexo & 1,546 & $0,528-4,526$ \\
\hline Cirugía previa & 0,35 & $0,074-1,647$ \\
\hline Insuficiencia renal & 1,442 & $0,416-5,003$ \\
\hline Insuficiencia cardíaca & 7,333 & $1,848-29,107$ \\
\hline Hipoalbuminemia & 1,574 & $0,491-5,049$ \\
\hline Neoadyuvancia & 1,113 & $0,122-10,162$ \\
\hline Anemia & 0,532 & $0,141-2,000$ \\
\hline Abordaje & 1,261 & $0,330-4,823$ \\
\hline $\begin{array}{l}\text { Anastomosis } \\
\text { (manual/mecánica) }\end{array}$ & 0,637 & $0,281-1,840$ \\
\hline Altura de la & & $0,587-6,069$ \\
anastomosis & 1,887 & $0,057-3,913$ \\
\hline Detransitación & 0,472 & $0,267-2,172$ \\
\hline Drenaje & 0,762 & $0,207-1,683$ \\
\hline $\begin{array}{l}\text { Experiencia del } \\
\text { cirujano }\end{array}$ & 0,590 &
\end{tabular}


agregarse además que la cirugía en manos de cirujanos jóvenes que no han completado su curva de aprendizaje se acompaña por lo general de un mayor tiempo operatorio.

Con respecto al tratamiento de la falla de sutura el primer problema que surge es, al igual que para su definición y alcance, estandarizar su manejo terapéutico. Para ello se han hecho múltiples intentos, como los del antes mencionado International Anastomotic Leak Study Group, ${ }^{18}$ el International Study Group of Rectal $\mathrm{Cancer}^{30} \mathrm{y}$ en estudios como el de Blumetti. ${ }^{31}$ En todo caso, se deben tener en cuenta los siguientes aspectos: momento de presentación (precoz vs. tardía), gravedad clínica, intra/extraperitoneal (con o sin detransitación para la última), grado de dehiscencia y de contaminación (cavidad libre vs. absceso). ${ }^{32}$ Sin perjuicio de ello pensamos que también deben considerarse aquí otros elementos clave como son la experiencia y el juicio del cirujano actuante y los recursos de los que dispone. Un paciente séptico, con un grado importante de contaminación abdómino-pélvica, tiene indicación formal de detransitación mediante la confección de una ostomía, probablemente asociada a la demolición o desmontaje de la anastomosis, ya que no suelen ser buenos candidatos a procedimientos de reparación o reconfección de la misma.

Si consideramos el momento de presentación, las fallas precoces requieren habitualmente una reintervención $\mathrm{du}-$ rante la primera semana del postoperatorio, cuando las adherencias inflamatorias aún son laxas y permiten hacerlo sin mayores inconvenientes. Sin embargo, la presentación tardía, en general como abscesos, posibilita un manejo menos agresivo, con técnicas mínimamente invasivas. En abscesos de hasta 3-4 cm, es factible la antibioticoterapia como único tratamiento ${ }^{33} \mathrm{y}$ en colecciones de mayor tamaño, el drenaje percutáneo ha demostrado buenos resultados también. ${ }^{6}$

Si se considera la altura de la anastomosis y la detransitación in situ o no, surgen distintas alternativas. La falla en cavidad libre (intraperitoneal) suele tratarse mediante desmontaje de la anastomosis y detransitación abocando ambos cabos (en anastomosis ileocólicas y colocólicas /colorrectales) o bien abandonando el cabo distal (Hartmann). Menos frecuentemente, la constatación de un defecto pequeño en general menor de $1 / 3$ de la circunferencia extrapolando los conceptos del trauma colorrectal, ${ }^{34}$ admite la reparación de la anastomosis asociada a detransitación proximal. En el caso de falla de suturas extraperitoneales, si tienen asociada una ostomía de detransitación y no presentan elementos locales o sistémicos de gravedad, pueden evaluarse periódicamente mediante estudio radiológico contrastado a la espera de resolución espontánea para luego cerrar la ostomía. De no ser posible, puede ser necesario reconfeccionarlas. Actualmente se encuen-

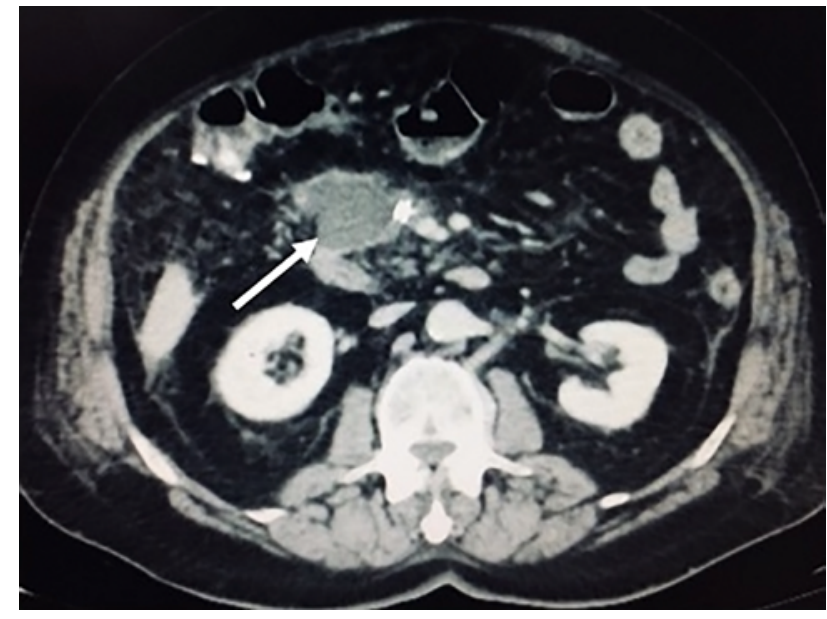

Figura 1: Absceso intraperitoneal (flecha) luego de colectomía derecha laparoscópica, manejo conservador. Fuente: autor.

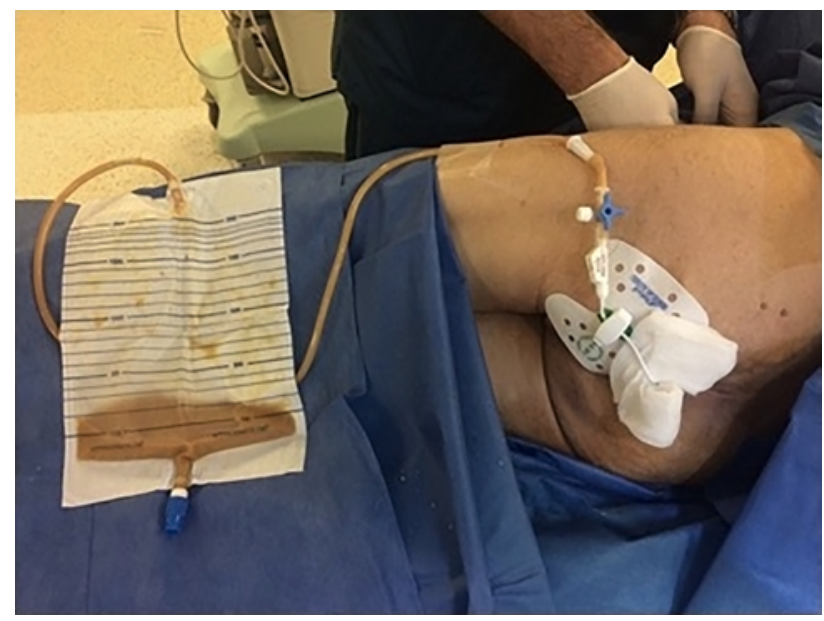

Figura 2: Absceso pelviano luego de resección anterior laparoscópica (drenaje percutáneo transglúteo). Fuente: autor

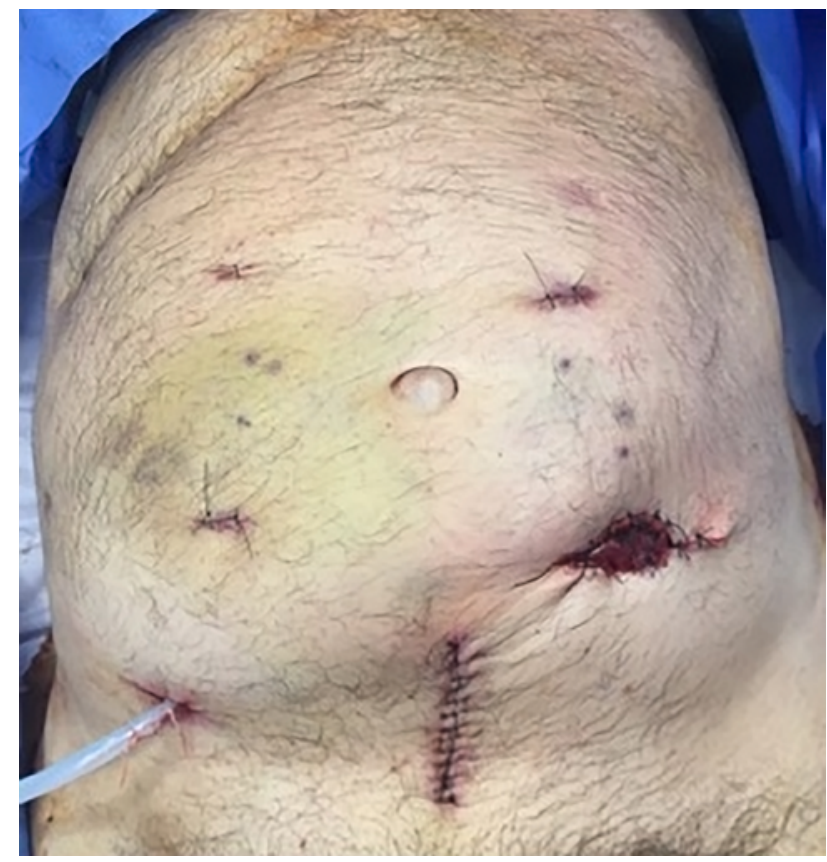

Figura 3: Reintervención laparoscópica con desmontaje y Hartmann. Fuente: autor. 
tra en fase de investigación el uso de esponjas conectadas a sistema aspirativo con el fin de reducir y cerrar la existencia de estos "senos ciegos" que persisten luego de la falla..$^{35}$ En nuestra serie se destaca en primer lugar el alto porcentaje de pacientes que se trataron mediante reintervención (85,7\%), y dentro de ese grupo también la gran proporción de casos en los que se optó como procedimiento definitivo por el desmontaje de la anastomosis (17/18 pacientes). En principio esto es concordante con el alto porcentaje de fallas tempranas observadas, en donde la vinculación con problemas técnicos hace más probable una reintervención quirúrgica en el postoperatorio inmediato. Por el contrario, aquellos pacientes con fallas tardías, habitualmente relacionadas a isquemia de los cabos o defectos pequeños, tienen mayores chances de obtener buenos resultados mediante tratamiento conservador o mínimamente invasivo, como también ha quedado demostrado en nuestra experiencia (figs. 1 y 2). Con respecto a la táctica empleada en los pacientes reintervenidos, el desmontaje de la anastomosis en contraposición a la reparación de la misma se justifica plenamente, dado que esta última conducta es muy riesgosa, sabiendo que en la mayoría de los casos solamente conducirá a aumentar el tamaño del defecto. ${ }^{36}$

En cuanto a la vía de abordaje para las reintervenciónes, casi todas fueron realizadas por laparotomía, excepto en 2 casos en que se realizó el desmontaje y Hartmann laparoscópico (fig. 3). Esto se debe fundamentalmente a la forma de presentación y la gravedad de los pacientes al momento de la cirugía (sepsis, acidosis, hemodinamia inestable, etc.). El tratamiento de esta complicación por vía laparoscópica sigue siendo controvertido, ya que a los factores anteriormente mencionados se agrega la complejidad en el manejo de asas delgadas distendidas, mayor sangrado y la necesidad de un debridamiento y toilette peritoneal exhaustivos a veces difícil de llevar a cabo por esta vía. ${ }^{37,38}$ Aun así, también existe evidencia que apoya la elección de un abordaje laparoscópico, sustentada sobre todo en el menor estrés sistémico y agresión parietal que produce..$^{39,40}$ En todo caso, es de suponer que a medida que los cirujanos ganen experiencia en el abordaje laparoscópico de la cirugía colorrectal, también lo harán en el manejo de sus complicaciones por esta misma vía.

La mortalidad global en la serie alcanzó el 15,2\% (17 pacientes), aunque solamente en 7 casos fue consecuencia directa de la falla de sutura. El resto de los casos fallecieron por otras causas, como progresión de su enfermedad, oclusión por bridas y patología cardiovascular.

Por último, debemos señalar las principales limitantes de este estudio. Algunas de ellas propias del carácter retrospectivo del mismo, lo que impide estandarizar aspectos preoperatorios (preparación colónica), intraoperatorios (experiencia del cirujano, técnicas, vías de abordaje, detransitación sistemática de anastomosis bajas) y determina la pérdida de datos en algunos casos. En segundo lugar y como fue analizado antes, el reducido tamaño muestral puede ser responsable de la ausencia de significación estadística en el análisis de algunos factores de riesgo estudiados por falta de potencia. Esto se debe a que ambas instituciones son consideradas como de bajo volumen.

\section{CONCLUSIONES}

La dehiscencia anastomótica constituye un desafío terapéutico en cirugía colorrectal y su manejo recae muchas veces en el juicio y experiencia del cirujano. En nuestra serie existió una incidencia algo elevada pero aun así dentro de lo aceptado según la literatura analizada. Asimismo, se encontró una asociación de riesgo estadísticamente significativa únicamente para el tamaño tumoral. El momento de presentación de la falla de sutura y el estado general del paciente son los que habitualmente determinan las posibilidades terapéuticas.

\section{BIBLIOGRAFÍA}

1. Makela JT, Kiviniemi H, Laitinen S. Risk factors for anastomotic leakage after left-sided colorectal resection with rectal anastomosis. Dis Colon Rectum 2003;46:653-60.

2. Miettinen RP, Laitinen SP, Makela JT, Paakkonen ME. Bowel preparation with oral polyethylene glycol electrolyte solution vs. no preparation in elective open colorectal surgery: prospective, randomized study. Dis Colon Rectum 2000;43:669-75.

3. Rickert A, Willeke F, Kienle P. Management and outcome of anastomotic leakage after colonic surgery. Colorectal Dis 2010;12: e216-23.

4. Walker KG, Bell SW, Rickard MJ. Anastomotic leakage is predictive of diminished survival after potentially curative resection for colorectal cancer. Ann Surg 2004;240:255-59.

5. Midura EF, Hanseman D, Davis BR. Risk factors and consequences of anastomotic leak after colectomy: a national analysis. Dis Colon
Rectum 2015;58:333-38.

6. Hyman N, Manchester TL, Osler T, Burns B, Cataldo PA. Anastomotic leaks after intestinal anastomosis: it's later than you think. Ann Surg 2007;245:254-58.

7. Bruce J, Krukowski ZH, Al-Khairy G, Russell EM, Park KGM. Systematic review of the definition and measurement of anastomotic leak after gastrointestinal surgery. Br J Surg 2001;88:1157- 68.

8. Kirchhoff P, Clavien PA, Hahnloser D. Complications in colorectal surgery: risk factors and preventive strategies. Patient Saf Surg 2010; 4:5.

9. Platell C, Barwood N, Dorfmann G, Makin G. The incidence of anastomotic leaks in patients undergoing colorectal surgery. Colorectal Dis 2007;9:71-79.

10. Kockerling F, Rose J, Schneider C, Scheidbach H, Scheuerlein H, Reymond MA, et al. Laparoscopic colorectal anastomosis: risk of 
postoperative leakage. Results of a multicenter study. Laparoscopic Colorectal Surgery Study Group (LCSSG). Surg Endosc 1999;13:639-44

11. Yamamoto S, Fujita S, Akasu T, Inada R, Moriya Y, Yamamoto S. Risk factors for anastomotic leakage after laparoscopic surgery for rectal cancer using a stapling technique. Surg Laparosc Endosc Percutan Tech 2012; 22:239-43.

12. Moghadamyeghaneh Z, Hanna MH, Alizadeh RF, Carmichael J, Mills S, Pigazzi A, et al. Contemporary management of anastomotic leak after colon surgery: assessing the need for reoperation. Am J Surg 2016; 211:1005-13.

13. Snijders HS, Wouters MW, van Leersum NJ. Meta-analysis of the risk for anastomotic leakage, the postoperative mortality caused by leakage in relation to the overall postoperative mortality. Eur J Surg Oncol 2012;38:1013-19.

14. Chadi SA, Fingerhut A, Berho M, DeMeester SR, Fleshman JW, Hyman NH, et al. Emerging trends in the etiology, prevention, and treatment of gastrointestinal anastomotic leakage. J Gastrointest Surg 2016; 20:2035-51.

15. Carlomagno N, Santangelo ML, Amato B, Calogero A, Saracco M, Cremone C, et al. Total colectomy for cancer: Analysis of factors linked to patients' age. Int J Surg 2014;12(Suppl 2):S135-39.

16. Bruce J, Krukowski ZH, Al-Khairy G, Russell EM, Park KGM. Systematic review of the definition and measurement of anastomotic leak after gastrointestinal surgery. Br J Surg 2001;88:1157- 68.

17. Rahbari NN, Weitz J, Hohenberger W, Heald RJ, Moran B, Ulrich A, et al. Definition and grading of anastomotic leakage following anterior resection of the rectum: a proposal by the International Study Group of Rectal Cancer. Surgery 2010;147:339-51.

18. Phitayakorn R, Delaney CP, Reynolds HL, Champagne BJ, Heriot AG, Neary P. International Anastomotic Leak Study Group. Standardized algorithms for management of anastomotic leaks and related abdominal and pelvic abscesses after colorectal surgery. World J Surg 2008;32:1147-56.

19. Floodeen H, Hallböök $O$, Rutegård J, Sjödahl R, Matthiessen $P$. Early and late symptomatic anastomotic leakage following low anterior resection of the rectum for cancer: are they different entities? Colorectal Dis 2013;15:334-40.

20. Lim SB, Yu CS, Kim CW, Yoon YS, Park IJ, Kim JC. Late anastomotic leakage after low anterior resection in rectal cancer patients: clinical characteristics and predisposing factors. Colorectal Dis 2016;18:135-40.

21. Morks AN, Ploeg RJ, Sijbrand Hofker H, Wiggers T, Havenga K. Late anastomotic leakage in colorectal surgery: a significant problem. Colorectal Dis 2013;15:271-75.

22. Grzona E, Quelin L, Solelo JM, Serafini V. Dehiscencias anastomóticas en coloproctología. Rev Argent Cirug 2018;110:13545.

23. Park JS, Choi GS, Kim SH, Kim HR, Kim NK, Lee KY, et al. Multicenter analysis of risk factors for anastomotic leakage after laparoscopic rectal cancer excision: the Korean laparoscopic colorectal surgery study group. Ann Surg 2013;257:665-71.

24. Yamamoto S, Fujita S, Akasu T, Inada R, Moriya Y, Yamamoto S. Risk factors for anastomotic leakage after laparoscopic surgery for rectal cancer using a stapling technique. Surg Laparosc Endosc
Percutan Tech 2012;22:239-43.

25. Zhu QL, Feng B, Lu AG, Wang ML, Hu WG, Li JW, et al. Laparoscopic low anterior resection for rectal carcinoma: complications and management in 132 consecutive patients. World J Gastroenterol 2010;16:4605-10.

26. Kawada K, Hasegawa S, Hida K, Hirai K, Okoshi K, Nomura A, et al. Risk factors for anastomotic leakage after laparoscopic low anterior resection with DST anastomosis. Surg Endosc 2014;28:2988-95.

27. Emile SH, Abd El-Hamed TM. Routine drainage of colorectal anastomoses: An evidence-based review of the current literature. Gastroenterol Res Pract 2017;2017:6253898.

28. Garfinkle R, Abou-Khalil J, Morin N, Ghitulescu G, Vasilevsky CA, Gordon $\mathrm{P}$, et al. Is there a role for oral antibiotic preparation alone before colorectal surgery? ACSNSQIP analysis by coarsened exact matching. Dis Colon Rectum 2017;60:729-37.

29. Kayano H, Okuda J, Tanaka K, Kondo K, Tanigawa N. Evaluation of the learning curve in laparoscopic low anterior resection for rectal cancer. Surg Endosc 2011;25:2972-79.

30. Sabbagh C, Maggiori L, Panis Y. Management of failed low colorectal and coloanal anastomosis. J Visceral Surg 2013;150:18187.

31. Blumetti J, Chaudhry V, Cintron JR. Management of anastomotic leak: lessons learned from a large colon and rectal surgery training program. World J Surg 2014;38:985-91.

32. Landmann, R. Surgical management of anastomotic leaks following colorectal surgery. Seminars in colon and rectal surgery $2014 ; 25$ : 58 66.

33. Kumar RR, Kim JT, Haukoos JS. Factors affecting the successful management of intra-abdominal abscesses with antibiotics and the need for percutaneous drainage. Dis Colon Rectum 2006;49:183-89.

34. Falcone RE, Wanamaker SR, Santanello SA, Carey LC. Colorectal trauma: primary repair or anastomosis with intracolonic bypass vs. ostomy. Dis Colon Rectum 1992;35:957-63.

35. Arezzo A, Verra M, Passera R, Bullano A, Rapetti L, Morino M. Long-term efficacy of endoscopic vacuum therapy for the treatment of colorectal anastomotic leaks. Dig Liver Dis 2015;47:342-45.

36. Agnifili, A., Schietroma, M., Carloni, A. The value of omentoplasty in protecting colorectal anastomosis from leakage. A prospective randomized study in 126 patients. Hepato-gastroenterology 2004;51:1694-97.

37. Sauerland S, Agresta F, Bergamaschi R, Borzellino G, Budzynski A, Champault $G$, et al. Laparoscopy for abdominal emergencies: Evidence-based guidelines of the European Association for Endoscopic Surgery. Surg Endosc 2006;20:14-29.

38. Vennix S, Abegg R, Bakker O, van den Boezem PB, Brokelman WJ, Sietses C, et al. Surgical re-interventions following colorectal surgery: Open versus laparoscopic management of anastomotic leakage. J Laparoendosc Adv Surg Tech A 2013;9:739-44.

39. Joh YG, Kim SH, Hahn KY, Stulberg J, Chung CS, Lee DK. Anastomotic leakage after laparoscopic protectomy can be managed by a minimally invasive approach. Dis Colon Rectum 2009;52:91-6.

40. Pera M, Delgado S, GarcÍa-Valdecasas JC, Pera M, Castells A, Pique' JM, et al. The management of leaking rectal anastomosis by minimally invasive techniques. Surg Endosc 2002;16:603-6.

\section{COMENTARIO}

El presente estudio plantea analizar los factores de riesgo y el manejo terapéutico de fallas Anastomóticas en resecciones colorrectales por neoplasias malignas con anastomosis primarias.

Sobre la totalidad de pacientes analizados (112), 50 cirugías fueron resecciones derechas, 48 resecciones izquierdas con anastomosis intraperitoneales y 18 resecciones colorrectales con anastomosis bajas. El abordaje fue VDLP en el 79\%. Las anastomosis fueron en un $68 \%$ con sutura mecánica. Se constató una filtración en el 17\% de pacientes (19 casos), de las cuales: 
- 10 (20\% de las ileocólicas, 52\% de las dehiscencias, $9 \%$ del total de pacientes).

- 5 (10,4\% de las colorrectales intraperitoneal, $26,3 \%$ de las dehiscencias, $4,5 \%$ del total).

- 4 (22,2\% de las colorrectales extraperitoneal, $21 \%$ de dehiscencias, $3,5 \%$ del total).

- $\quad$ E1 68\% de las dehiscencias se presentaron antes del sexto día postoperatorio.

En cuanto al manejo y tratamiento en el $85 \%$ se procede a desarmar y ostomizar.

La dehiscencia anastomótica es una de las complicaciones más temidas en la cirugía colorrectal. Es una condición que tiene alta morbi-mortalidad y genera una situación desagradable tanto para el médico, como para la familia y el paciente.

Lo ideal es reducir al máximo esta posibilidad, para ello hay que tomar muchos recaudos:

- Asegurarse la adecuada irrigación de los cabos anastomóticos.

- Anastomosis sin tensión.

- Suturas mecánicas adecuadas.

- Sutura manual con buena técnica.

A pesar de todo esto, el riesgo existe y los factores que lo aumentan son aquellos pacientes con enfermedad tumoral avanzada, anemia, hipoalbuminemia, obesidad, antecedente de neoadyuvancia, y altura de la anastomosis con respecto al margen anal.

Por tal motivo, cuando se produce una conjunción de estos factores de riesgo, hay que tener en cuenta proteger esa anastomosis con un ostoma proximal.

Es preferible un ostoma de protección, que arriesgar a una filtración anastomótica con la consecuente reoperación y desarme de esa anastomosis compleja o ultrabaja; que probablemente, si supera la complicación, lo condene al paciente a un ostoma permanente.

Es cierto que esta complicación conlleva un alto costo hospitalario, pero también el costo es para el paciente y el grupo quirúrgico actuante. Es por ello que debemos insistir en una minuciosa técnica quirúrgica y utilizar las maniobras de pruebas intraoperatorias (algunas más básicas y otras más tecnificadas y costosas) que nos permitan corroborar que esa anastomosis está en óptimas condiciones.

Marcelo Pollastri

Centro de Coloproctología y Enfermedades Digestivas. Rosario, Santa Fe. 